\title{
Review Article \\ Rice Breeding for High Grain Yield under Drought: A Strategic Solution to a Complex Problem
}

\author{
Shalabh Dixit, Anshuman Singh, and Arvind Kumar \\ International Rice Research Institute, DAPO Box 7777, Metro Manila, Philippines \\ Correspondence should be addressed to Arvind Kumar; a.kumar@irri.org
}

Received 8 August 2013; Revised 3 November 2013; Accepted 11 March 2014; Published 28 April 2014

Academic Editor: Kent Burkey

Copyright ( 2014 Shalabh Dixit et al. This is an open access article distributed under the Creative Commons Attribution License, which permits unrestricted use, distribution, and reproduction in any medium, provided the original work is properly cited.

\begin{abstract}
Drought is one of the major abiotic stresses that affect rice production in rainfed areas. Recent trends in climate change have predicted a further increase in drought intensity, making the development of new drought-tolerant rice cultivars critical to sustain rice production in this ecosystem. The use of grain yield as a selection criterion at the International Rice Research Institute (IRRI), through proper population development and precise phenotyping techniques, has allowed the development of several high-yielding rice cultivars that have been released in major rainfed rice-growing areas. This strategy has also allowed the identification of several major quantitative trait loci (QTLs) that show large effects under drought across environments and genetic backgrounds. These QTLs are being pyramided together to develop drought-tolerant versions of popular drought-susceptible varieties. The nearisogenic lines (NILs) developed can replace the popular, high-yielding but drought-susceptible varieties in rainfed areas prone to drought. Additionally, these NILs serve as suitable genetic material for the study of molecular and physiological mechanisms underlying these QTLs. This may provide a better understanding of plant functions responsible for high grain yield under drought and lead to the identification of new traits and genes.
\end{abstract}

\section{Introduction}

Climate change threatens the sustainability of modern-day agriculture. Constantly changing climatic conditions around the world demand constant efforts to understand and adapt to environmental challenges for sustainable crop production. The challenge is even greater for crops such as rice (Oryza sativa L.), which is the staple of more than half the world's population and grown under diverse environmental conditions. In 2012, 158.4 million ha around the world was devoted to rice cultivation, with a production of around 697.2 million http://www.irri.org/. Majority of the rice produce comes from developing countries in Asia, Africa, and Latin America. While these areas contribute to a major share of world rice production, a large proportion of rice in these areas is grown by small and marginal farmers with minimum inputs and infrastructure. In the absence of basic infrastructure such as irrigation facilities, many areas rely on rainfall and are severely affected in cases of uneven rainfall incidences, leading to drought and/or flood. At least 30.9\% of the total rice area around the world is rainfed (Figure 1) and is subjected to variable intensities of drought and flood. Drought is one of the major abiotic stresses that lead to a decline in rice production in the rainfed areas. In Asia alone, a total of 23 million ha of rice area is affected by drought of variable intensities. Apart from this, recent climate change trends point to increasing water deficit in the coming years [1]. This may lead to a further increase in the intensity and frequency of drought. Even areas with assured irrigation facilities face issues related to declining availability of irrigation water (Figure 2), the result of declining fresh water resources, and increasing demand from industrial sectors and urban centers [2]. In the coming sections of this paper, we try to summarize the nature of target environments (TE) highly prone to drought stress. The paper briefly gives an overview of the nature of grain yield under drought and its suitability as a selection criterion. A detailed review of activities related to breeding rice for drought tolerance using grain yield as a selection criterion at IRRI is presented. The paper focuses on the methodologies used to develop new drought-tolerant varieties, the varieties released, and the quantitative trait loci (QTLs) identified for yield under drought. The paper also 


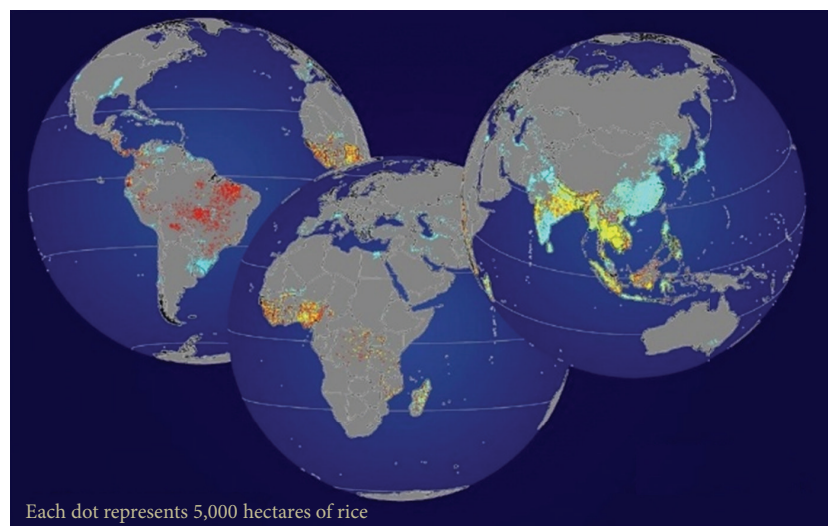

- Irrigated fields

- Rainfed, flooded fields

- Rainfed, nonflooded fields

FIGURE 1: Rice area occupied by irrigated, rainfed upland, and rainfed lowland ecosystems around the world. Source: Hijmans [38].

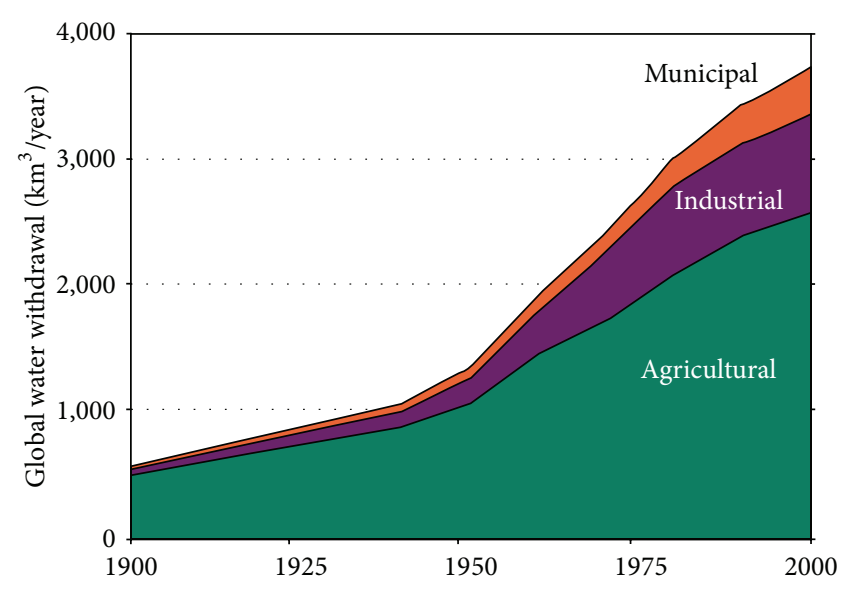

FIGURE 2: Increasing sectoral competition for blue water withdrawals for human uses. Source: Molden et al. [2].

summarizes the effects of these QTLs on other traits that contribute to drought tolerance in rice and discusses ongoing activities in this field and future perspective.

\section{The Target Environment}

Rice is a semiaquatic crop and the only cereal that can be grown in an anaerobic environment. However, the large acreage of rice around the world has led to its cultivation in diverse ecosystems such as rainfed uplands and lowlands, irrigated, and deep water ecosystems. All these ecosystems, to variable extent, face the threat posed by climate change. However, conditions in rainfed environments are particularly unstable. In general, rainfed environments may classified into four different types based on their presence on the toposequence. This includes rainfed uplands, rainfed shallow lowlands, rainfed medium lowlands, and deep water ecosystems. All these ecosystems are characterized by specific scenarios of stresses related to water. Rainfed uplands are the most severely affected by drought stress. This is mainly due to the lighter soil texture and quick drainage of rain water due to their presence in the uppermost part of the topo-sequence. In general, early to medium duration varieties are grown in these areas and practices like dry and wet direct seeding are the more common in this ecosystem as compared to the others. The second most drought affected ecosystem is the rainfed shallow lowland. Mostly characterized by slightly heavier soil texture, both direct seeded and transplanted rice are grown in this ecosystem. Medium to late duration varieties are normally preferred in these areas. The next in topo-sequence are the rainfed medium lowlands. These are ecosystems prone to both drought and submergence. High intensity of rainfall in one part of the season causes flash floods in these areas, while the crop suffers from drought the rest of the season due to prolonged dry spells. Normally, direct seeding or transplanting in puddled soil is practiced and medium to late duration rice varieties are cultivated in these ecosystems. The deepwater ecosystems constitute the lowest part of the topo-sequence. These areas are normally situated near river basins and deltas. Being in the lowest level of the topo-sequence, these areas are highly prone to short and long duration flooding and also to salinity (in case of coastal areas and deltas). The absence of irrigation and the unpredictable nature of rain water in these areas lead to untimely farm operations, a common scenario in the case of delayed onset of the rainy season or long dry spells during the latter part of the cropping season. The high risk associated with farming in areas prone to drought stress may make farmers reluctant to supply adequate seed-fertilizer inputs to the crop which could increase profitability in normal years but lead to a loss of capital investment in poor years [3]. It is also observed that apart from direct losses in income and production, drought also leads to loss in income in normal years [4]. Apart from this, the abiotic stresses also make the rice crop more vulnerable to biotic stresses, leading to further decline in crop production. For example, there are more diseases (e.g., rice blast and brown spot) in drought-infested fields than in fields with proper water supply. Drought is the predominant abiotic stress that affects crop production in the rainfed environment. Dry spells at the reproductive stage are particularly damaging as the crop is most prone to yield loss at this stage $[5,6]$. Developing drought tolerant varieties is one of the possible solutions to increase rice production in these areas. Despite these facts, majority of the rainfed areas are planted with high-yielding varieties preferred by farmers and consumers. These varieties were not selected for tolerance for stresses such as drought and farmers face severe crop losses in the event of a drought $[7,8]$. For example, high yielding rice varieties like IR64, Swarna, TDK1, and Sabitri are some of the major varieties which cover a large area under rainfed conditions in eastern India, Bangladesh, Lao PDR, and Nepal. The studies conducted at IRRI show the severe effect of drought on yield of these varieties $[8,9]$. On the other hand, several valuable traditional genotypes have evolved in these regions with adaptation to specific environmental challenges, but they are not used for commercial cultivation because of poor yield potential and undesirable morphological and grain characteristics. The complex interactions of biotic and 
abiotic stresses in these rainfed environments demand the development of varieties with tolerance for multiple stresses, along with high yield potential and preferred grain quality. Suitable breeding programs involving location-specific preferred varieties and landraces may provide the way to sustain rice production in unfavourable environments.

\section{Breeding Rice for Drought Tolerance}

The requirements of drought tolerant rice varieties have long been felt to ensure crop production in rainfed areas. Being a semiaquatic crop, rice gets severely affected by even moderate intensities of drought and varying levels of yield decline can be seen depending on the variety being grown. Drought tolerance can broadly be achieved through three major mechanisms. (1) Drought escape due to early completion of the life cycle: this is particularly useful in the scenario where drought is a recurrent phenomenon at the end of the growing season. In such cases, early flowering varieties can escape terminal drought. (2) Dehydration avoidance which enables the plant to uptake or conserve more water to avoid dehydration: this is achieved through traits related to root architecture, stomatal control, and transpiration efficiency. (3) Dehydration tolerance which is achieved through traits such as cell membrane stability, osmotic adjustment, stem reserve mobilization, and stability of flowering process: conventional and molecular breeding approaches targeting these traits have been used in the past to develop drought tolerant rice varieties. It has been reported that the progress in breeding for drought tolerance has been slow [10]. One of the reasons for this is the complexity of genetic control of grain yield under drought. One or more of the traits mentioned above along with traits related to yield potential such as number of tillers, panicles, and fertile grains can play a role in determining the yield under drought. Low yield potential under nonstress conditions has also been known as another setback for several drought breeding programs which may have achieved high tolerance to drought in the lines but fail to provide high yield under nonstress conditions, leading to difficulty in release and/or adaptation of the lines. While selection for all these traits becomes difficult simultaneously, using grain yield per se as a selection criterion in a large population segregating for these traits can be an efficient alternative. Similar to conventional breeding programs, a large number of traits have been targeted for identification of QTLs under drought. Kamoshita et al. [11] compiled a literature review of studies targeting different classes of traits related to drought tolerance in rice (see Supplementary Table 1 at Supplementary Material available online at http://dx.doi.org/10.1155/2014/863683). Despite availability of such a large number of traits affecting a variety of drought related traits and some of these QTLs being able to explain a large proportion of phenotypic variance for the traits, very few reports of their utilization for marker assisted selection (MAS) exist. The possible reasons for this could be the inconsistency of QTLs across varying environments and the lack of high effect on grain yield despite the large effect on the target secondary traits. Table 1 presents a few examples of QTLs that were used for MAS in rice (as reviewed by
Ashraf [12]). MAS programs targeting multiple QTLs related drought tolerance as well as that targeting grain yield per se seem to be more effective in enhancing the yield under drought. Those targeting one QTL related to a secondary trait were able to improve the related traits but had less effect on grain yield under drought. While several conventional and molecular breeding programs around the world continue to make their efforts to improve rice for drought tolerance, incorporating grain yield per se in these programs could be an efficient way to improve the effect of these programs.

\section{Grain Yield under Drought: Variability Available, Choice of Parents, and Suitability as Selection Criterion}

The success of a breeding program depends on the availability of genetic variability in the population subjected to selection, the selection criterion, and availability of suitable selection protocol. The choice of suitable parents based on targets set by the breeding program plays a crucial role in the successful development of populations. Development of large breeding populations using crosses between drought-tolerant landraces and high-yielding drought-susceptible varieties has proven effective in combining high yield potential with drought tolerance [13]. Apart from this, the use of popular varieties as recipient parents in the target environment also provides a chance to develop lines with desired traits related to grain quality, plant type, tolerance for other stresses in a particular region, and farmers' preferences, thereby increasing the probability of adoption of the new varieties. It is also advantageous to choose landraces that may have tolerance for other stresses along with drought tolerance to increase the possibility of coming up with lines that can tolerate multiple stresses. Recent studies at IRRI have used drought tolerant donors (N22, Dagad Deshi, Moroberekan, Aus 276, Vandana, Apo, and IR55419-04) to develop large populations by crossing them with high-yielding popular varieties from different countries (IR64, Swarna, TDK1, MTU1010, Sambha Mahshuri, Sabitri) and screening them for grain yield under reproductive-stage drought and irrigated control situations. It is also important that the parents are selected on the basis of the target ecosystem. This becomes important because it has been seen that, in rice, the plant types required for upland and lowland conditions are very distinct. The plant types suitable for flooded lowland conditions are hightillering, medium-to- long-duration dwarf plant types with drought tolerance. This kind of plant type ensures high yield under normal conditions with minimum threat to lodging. The long maturity duration also allows the plant to attain high biomass and high tillering which in turn leads to an increase in yield under normal conditions. The added tolerance to drought in such lines also ensures yield under drought conditions. Majority of the popular varieties for the lowland ecosystem such as Swarna, IR64, TDK1 etc. show these morphological characters. However, they need to be improved for tolerance to drought. In contrast to the lowland ecosystem, the plant types suitable for upland conditions are semidwarf to semitall, early-to-medium-duration lines 
TABLE 1: Some examples of enhancing drought tolerance of rice varieties through marker assisted selection (MAS).

\begin{tabular}{|c|c|c|c|c|c|}
\hline QTL used & $\begin{array}{l}\text { QTL donor } \\
\text { line/cultivar }\end{array}$ & $\begin{array}{c}\text { Recipient } \\
\text { line/cultivar }\end{array}$ & $\begin{array}{l}\text { Line/cultivar } \\
\text { developed }\end{array}$ & Trait improved & Reference \\
\hline $\begin{array}{l}\text { QTL9 (on } \\
\text { chromosome 9) }\end{array}$ & Azucena & Kalinga III & $\begin{array}{l}\text { Near-isogenic } \\
\text { lines (NILs) }\end{array}$ & $\begin{array}{l}\text { Improved root length and thickness under both } \\
\text { irrigated and drought-stressed field conditions }\end{array}$ & Steele et al. [35] \\
\hline $\begin{array}{l}\text { QTL9 (on } \\
\text { chromosome 9) }\end{array}$ & Azucena & Kalinga III & $\begin{array}{l}\text { Near-isogenic } \\
\text { lines (NILs) }\end{array}$ & $\begin{array}{c}\text { Under field conditions, NILs out-performed } \\
\text { Kalinga III for grain and straw yield. However, the } \\
\text { lines had higher straw yield than grain yield as } \\
\text { introgressed genes involved in partitioning of } \\
\text { biomass to the roots and stems, rather than to the } \\
\text { grain }\end{array}$ & Steele et al. [36] \\
\hline $\begin{array}{l}\text { QTL2 (on } \\
\text { chromosome 2) QTL9 } \\
\text { (on chromosome 9) } \\
\text { and QTL11 (on } \\
\text { chromosome 11) }\end{array}$ & Azucena & Kalinga III & $\begin{array}{c}\text { Birsa Vikas } \\
\text { Dhan } 111 \text { (PY } \\
84)\end{array}$ & $\begin{array}{l}\text { Early maturing and high yielding; good grain } \\
\text { quality; drought tolerant }\end{array}$ & Steele [37] \\
\hline qtl12.1 & Way Rarem & Vandana & $\begin{array}{l}\mathrm{F}_{3} \text {-derived } \\
\text { populations }\end{array}$ & $\begin{array}{c}\text { Improved biomass production; panicle number; } \\
\text { and } 47 \% \text { average yield }\end{array}$ & $\begin{array}{l}\text { Bernier et al. } \\
{[21]}\end{array}$ \\
\hline
\end{tabular}

Source: Ashraf [12].

(as seen in popular varieties, IR74371-54-1-1, Apo, IR5541904 , etc.) with a comparatively higher degree of drought tolerance due to the more frequent and severe drought events in that environment. Screening large breeding populations across a wide range of target environments under naturally occurring drought stress is difficult, expensive, and risky. The unpredictable nature of rainfall across different seasons makes it difficult to achieve similar intensities of drought in all environments to identify and select the best suited breeding material [14]. Screening these populations under reproductive-stage drought in the dry season in replicated trials conducted under managed water stress conditions has proven advantageous. Moderate to high correlations have been reported between the performance of lines during the dry-season screening at IRRI and the wet-season drought in India [15]. Another important factor on which the success of a breeding program depends is the availability of a suitable selection criterion. The efficiency of a trait as a selection criterion is determined by its repeatability or broad-sense heritability $(\mathrm{H})$ [16]. It has long been believed that grain yield under drought has low $\mathrm{H}$ values due to the highly complex genetic control of the trait and high genotype $\mathrm{X}$ environment (GXE) interaction associated with this trait. However, recent studies at IRRI have reported moderate to high $\mathrm{H}$ for grain yield under reproductive-stage drought stress with precise screening protocols and use of large breeding populations. A positive response of direct selection for grain yield under severe stress conditions has been reported under similar stress levels in upland and lowland areas in these studies [17]. For example, Kumar et al. [7] have reported a positive response of selection of lines under severe stress trials across upland and lowland ecosystems at IRRI and in eastern India, respectively. Similarly, Venuprasad et al. [18] reported higher yield gain in lines selected under severe stress conditions as compared with those selected under non-stress conditions. Apart from developing lines with higher grain yield under drought, it is also important that these lines have yield potential equal to or more than that of popular high-yielding varieties. This is crucial in ensuring high yield in favourable years, which affects adoption of a variety after its release. Several IRRI studies, which involved breeding or mapping populations derived from a drought-tolerant cultivar and a high-yielding variety, have shown the presence of transgressive segregants with higher yield as compared with parents under both stress and nonstress conditions [17]. This also shows that grain yield under drought is not only a function of drought tolerance of the lines but also of yield potential, which relates to characters such as high tillering ability and more panicles and grains per plant. This is probably one of the reasons most traditional drought tolerance donors do not do well under nonstress conditions or under mild stress conditions where yield potential becomes as important as tolerance for drought in maintaining yield levels. These findings have shown the importance and suitability of direct selection for grain yield under drought stress and nonstress conditions in combining high yield potential with drought tolerance.

\section{Breeding High Yielding Drought Tolerant Lines with Multiple Stress Tolerance}

The suitability of grain yield as selection criterion has opened the way to the initiation of large-scale breeding programs for drought tolerance. Based on the development and screening of large segregating populations under drought and irrigated conditions, rice varieties with high yield potential and good yield under drought may evolve. Figure 3 presents a standard workflow for a conventional breeding program used at IRRI to develop high-yielding drought-tolerant rice varieties with tolerance for major biotic stresses such as bacterial leaf blight and rice blast. $\mathrm{F}_{1}$ plants are developed by crossing a high-yielding variety and a drought-tolerant cultivar. Some of these drought-tolerant cultivars or drought-susceptible high-yielding varieties are donors of tolerance for other biotic or abiotic stresses. It is more advantageous to choose 


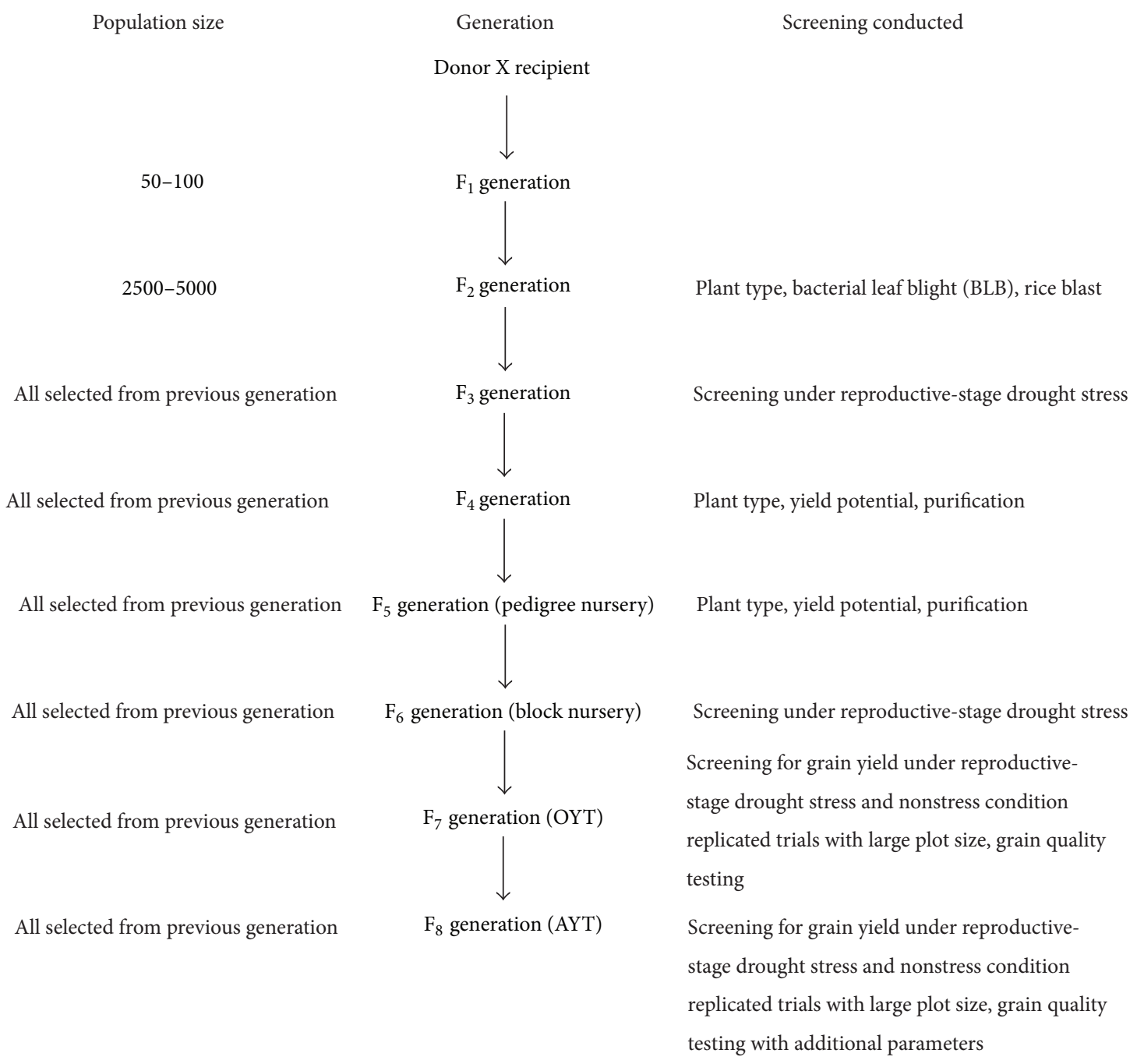

FIGURE 3: Standard flow of material used at IRRI in a conventional breeding program to develop high-yielding drought-tolerant lines.

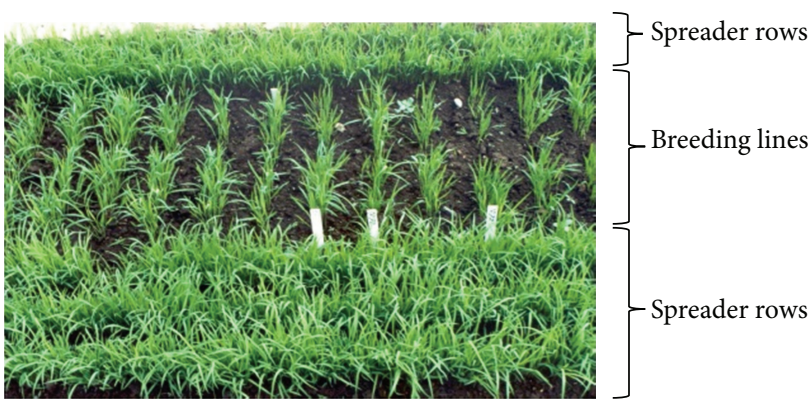

(a)

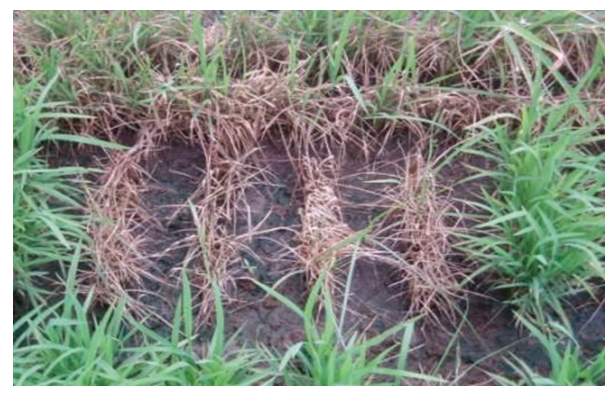

(b)

FIGURE 4: (a) Layout of breeding lines and spreader rows at IRRI blast nursery to screen for blast resistance at the nursery stage. (b) Breeding lines showing different tolerance reactions to rice blast.

such cultivars so that tolerance for multiple stresses can be achieved in the lines. An $F_{1}$ population of 50-100 plants per cross is considered desirable. This $\mathrm{F}_{1}$ population is selfed to develop a large $F_{2}$ population. Seeds of this population are planted in nursery beds where they are inoculated by natural inoculum with three to four lineages of Magnaporthe oryzae (Figure 4). The plants are scored for tolerance to the strains based on standard evaluation system (SES) [19]. Lines with scores between 0 and 4 are advanced further to the field where they are grown under well-watered conditions. A population 


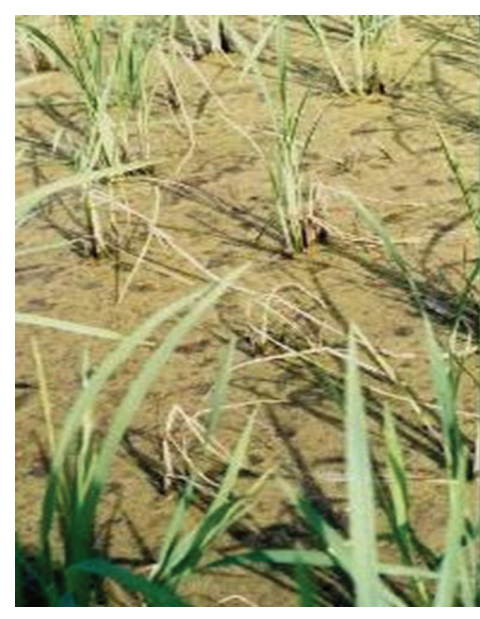

(a)

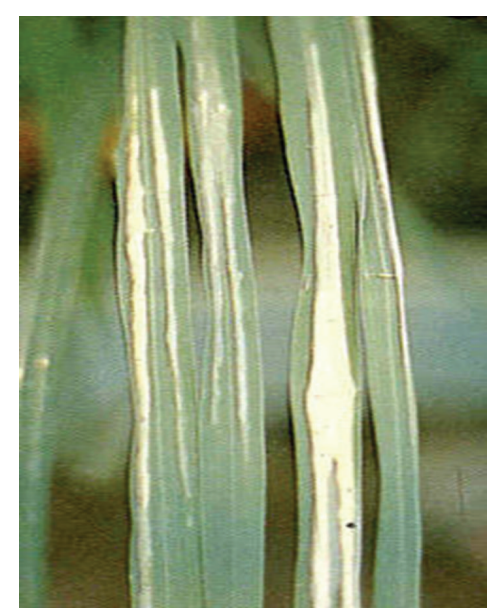

(b)

Figure 5: (a) Plants with different levels of tolerance for bacterial leaf blight (BLB) in the field. (b) Rice leaves showing symptoms of BLB.

TABLE 2: Selection criterion used at IRRI to identify suitable plants in early-generation $\left(\mathrm{F}_{2}\right)$ breeding materials.

\begin{tabular}{|c|c|c|}
\hline Criterion & Lowland & Upland \\
\hline Plant type & Erect, compact, high-tillering & Erect, compact, high-tillering \\
\hline \multirow{2}{*}{ Maturity (DAS) } & Medium: $110-120$ & Early: $\leq 100$ \\
\hline & Late: $121-140$ & Medium: 101-110 \\
\hline Height $(\mathrm{cm})$ & $100-120$ & $100-130$ \\
\hline Visual yield & Good & Good \\
\hline \multirow{2}{*}{ Grain shape ${ }^{*}$} & Long slender & Medium slender \\
\hline & $\begin{array}{l}\text { Medium cylinder } \\
\text { Medium bold }\end{array}$ & Medium bold \\
\hline Disease resistance & Resistant to blast and BLB & Resistant to blast and brown spot \\
\hline Insect resistance & Brown plant hopper, gall midge & Brown plant hopper, gall midge \\
\hline
\end{tabular}

${ }^{*}$ Selection for grain shape may vary according to target environment.

size of 2500-5000 is considered optimum in this generation. Inoculation for bacterial leaf blight with PXO61 and PXO86, two strains of Xanthomonas oryzae pv., oryzae is done in the field around 30 days after transplanting (Figure 5). Plants showing scores above 3 based on SES are rejected. Singleplant selection is carried out in this generation based on the criterion presented in Table 2. The major focus at this stage is selecting plants with good plant type and suitable maturity duration based on target ecosystem and preferred grain type. Selections for dry direct seeded upland conditions are separated from those made for transplanted lowland conditions at this stage and are advanced to the $\mathrm{F}_{3}$ generation in their respective environments. The $\mathrm{F}_{3}$ population developed from selected plants in the $F_{2}$ is screened under drought stress conditions and subjected to selection for tolerance for drought. $\mathrm{F}_{3}$ lines are planted in single-row plots for the respective set of material in upland and lowland conditions. Plant selections are made in lowland conditions, while panicles are selected in upland conditions due to continuous seeding in this ecosystem. The selected plants/panicles are advanced to the $\mathrm{F}_{4}$ generation and planted under nonstress conditions in single-row plots. Selections from these trials are advanced to the $\mathrm{F}_{5}$ generation and the same procedure of screening the plants under drought is repeated. In the $\mathrm{F}_{6}$ generation, lines are screened under both drought stress and nonstress conditions in the lowland ecosystem and in the nonstress ecosystem in the upland because of the limited amount of seeds harvested from selected panicles. Lines with high yield under both conditions are advanced to the observational yield trial (OYT). All trials conducted until the $\mathrm{F}_{6}$ generation are unreplicated. At this stage, lines are divided into groups based on crop duration and lines belonging to each group are planted with checks in replicated yield trials in $\alpha$-lattice design in larger plots. The harvest from this trial is also subjected to grain quality testing for traits such as amylose content, gelatinization temperature, and chalkiness. Lines selected from this trial constitute the advanced yield trial (AYT). These trials are conducted in replicated $\alpha$-lattice design with large plot sizes. Grain quality tests are repeated in this generation with some quality traits added (e.g., milling yield recovery). Pure seeds harvested from these trials are also used in similar experiments in the target environment to evaluate the performance of these lines in these conditions. This approach allows the use of suitable 
donors for incorporating tolerance for multiple stresses along with identification of lines with high-quality grains. Several varieties developed through this procedure are being released in South and Southeast Asia (Table 3). Line IR74371-70-1-1 was released under three different names in three countries in South Asia: India, Bangladesh, and Nepal. This indicates the suitability of grain yield as a selection criterion to identify lines showing a yield advantage across a wide range of environments. The performance of these lines also shows that, regardless of complexity of traits such as yield under drought, it is possible to develop genetic material with lower GXE interactions in rice through suitable breeding procedures. Precise phenotyping techniques that involve screening of varieties for biotic and abiotic stress tolerance within the same generation can be highly beneficial in rapidly developing varieties tolerant to multiple stresses. For example, screening for rice blast at the seedling stage, bacterial leaf blight at the vegetative stage, and drought at the reproductive stage of the crop can be done in one generation. Similarly, lines can be screened for tolerance for submergence at the seedling stage and tolerance for drought at the reproductive stage. This strategy not only allows the screening of lines for multiple stresses but also facilitates the identification of lines that can tolerate these stresses when they simultaneously occur within the same season. The success of this breeding strategy with bi-parental crosses also suggests its suitability for handling populations developed through multiple crosses. However, in those cases larger population sizes at $\mathrm{F}_{1}$ generation are needed to be developed in order to allow enough recombination and genetic variability in the population. Subsequently, the $\mathrm{F}_{2}$ population size must also be increased. Based on the choice of parents, screening for other traits like disease tolerance (BLB, Blast or others), insect tolerance (brown plant hopper, gall midge, etc.), or tolerance to other abiotic stresses (temperature, submergence, salinity, etc.) can be conducted at different generations during the development of fixed lines. While the basic procedure and principle remain the same, the use of multiple parents can provide higher genetic variability in terms of tolerance to stresses, yield, and grain quality.

\section{QTLs for High Grain Yield under Drought}

Several varieties have been released by the use of conventional breeding approaches using grain yield as a selection criterion. However, these varieties are new breeding lines which possess new phenotypic characteristics. As discussed in the previous sections, large areas covered by high yielding drought susceptible varieties need to be targeted. Identification and introgression of large effect QTLs for grain yield under drought could be one of the ways to make large-scale impact on these areas. Drought tolerant versions of popular mega varieties such as IR64 and Swarna may replace these varieties easily in rainfed areas where these varieties are already preferred due to their yield potential and grain quality. This necessitates a need of identification of QTLs for higher grain yield under drought. The phenotyping techniques used to develop varieties through selection under drought stress and nonstress conditions can also be used to phenotype large mapping populations. Different kinds of mapping populations such as recombinant inbred lines (RILs), backcross inbred lines (BILs), or doubled haploid lines (DHLs) can be developed and used for mapping QTLs under drought [20]. In particular, BILs provide an advantage in the identification of QTLs and subsequent development of NILs. For example, development of $\mathrm{BC}_{2} \mathrm{~F}_{3}$-derived lines ensures a higher percentage of high-yielding recipient parents in the genetic background, which allows achieving better plant types in the population. These populations contain lines with high background recovery, thus leading to droughttolerant lines with morphology similar to that of the recipient. These lines can either be used directly as introgression lines or can be used for easy development of NILs (maintenance breeding). In contrast, these populations also indicate the presence of some lines with a higher percentage of the donor genome. However, some of these lines show promising characteristics in terms of yield potential, grain type, and plant morphology. The lines can be purified and deployed directly for testing and release. Such plants can be screened for the presence of QTLs identified in the population and used for direct testing in target environments or they can be used as parents for further backcross programs. It is much easier to recover a high percentage of recipient background with the use of such lines compared with RILs of $\mathrm{F}_{3}$ derived lines, which contain a higher percentage of the donor genome.

6.1. Phenotyping of Mapping Populations. Precise phenotyping of mapping populations depends on three major factors: experimental design, crop management, and uniformity in drought stress across the trial. Trials must be conducted with large populations in order to have a more precise estimation of the QTLs. A population size of 350-400 lines is optimum for these experiments. The population must be planted in replicated trials with two to three replications. Figures 6(a) and 6(b) show field trials conducted under transplanted lowland conditions and dry direct seeded upland conditions, respectively. Larger plots provide a better estimation of plot yield; however, plot size must be kept to a minimum of $1 \mathrm{~m}^{2}$. Trials can be conducted in two or more replications; however, having three replications is more advantageous for precise estimation of trait means. Large replication size due to the large number of lines within each replication also demands the use of suitable experimental design. The $\alpha$-lattice design is found to be suitable for such experiments as it reduces error due to large replication size as compared with designs such as randomized complete block design (RCBD). The design divides large replications into smaller blocks and the effect of blocks within the replications is accounted for in the analytical models. Stress is initiated at 50 DAS under lowland and 45 DAS under direct-seeded upland conditions. Perforated PVC pipes can be installed to measure daily decline in water table depth after stress initiation under lowland conditions. Under upland condition, tensiometers can be installed to measure soil moisture potential. Trials should be rewatered when the water table under lowland condition falls below $100 \mathrm{~cm}$ while under upland conditions, trials can be re-watered when the tensiometer readings are $\geq 50$. In both ecosystems, rewatering is also based on the percentage 
TABLE 3: Varieties developed at IRRI through conventional breeding approaches involving direct selection for grain yield under drought.

\begin{tabular}{|c|c|c|c|c|c|c|}
\hline Name & Designation & $\begin{array}{l}\text { Days to } \\
\text { maturity }\end{array}$ & $\begin{array}{c}\text { Plant height } \\
(\mathrm{cm})\end{array}$ & Country & Ecosystem & $\begin{array}{c}\text { Release } \\
\text { year }\end{array}$ \\
\hline Sahod Ulan 1 & IR74371-54-1-1 & 110 & 104 & Philippines & RL, UP & 2009 \\
\hline Hardinath 1 & IR80411-B-49-1-1 & 115 & 100 & Nepal & RL & 2009 \\
\hline Sahbhagi dhan & IR74371-70-1-1 & 110 & 104 & India & RL, UP & 2010 \\
\hline BRRI dhan 56 & IR74371-70-1-1 & 110 & 108 & Bangladesh & $\mathrm{RL}$ & 2011 \\
\hline Sookha dhan 3 & IR74371-70-1-1 & 110 & 108 & Nepal & $\mathrm{RL}$ & 2011 \\
\hline Sookha dhan 1 & IR74371-46-1-1 & 110 & 101 & Nepal & $\mathrm{RL}$ & 2011 \\
\hline Sookha dhan 2 & IR74371-54-1-1 & 110 & 104 & Nepal & $\mathrm{RL}$ & 2011 \\
\hline Katihan 1 & IR79913-B-176-B-4 & 105 & 90 & Philippines & UP & 2011 \\
\hline Sahod Ulan 3 & IR81412-B-B-82-1 & 120 & 107 & Philippines & $\mathrm{RL}$ & 2011 \\
\hline Sahod Ulan 5 & IR81023-B-116-1-2 & 115 & 130 & Philippines & $\mathrm{RL}$ & 2011 \\
\hline Sahod Ulan 6 & IR72667-16-1-B-B-3 & 115 & 100 & Philippines & $\mathrm{RL}$ & 2011 \\
\hline Sahod Ulan 8 & IR74963-262-5-1-3-3 & 125 & 100 & Philippines & $\mathrm{RL}$ & 2011 \\
\hline Inpago LIPI Go 1 & IR79971-B-191-B-B & 110 & 115 & Indonesia & UP & 2011 \\
\hline Inpago LIPI Go 2 & IR79971-B-227-B-B & 113 & 114 & Indonesia & UP & 2011 \\
\hline
\end{tabular}

UP: rainfed upland, RL: rainfed lowland.

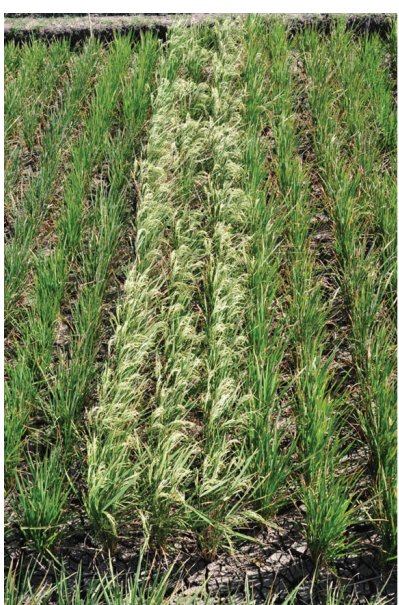

(a)

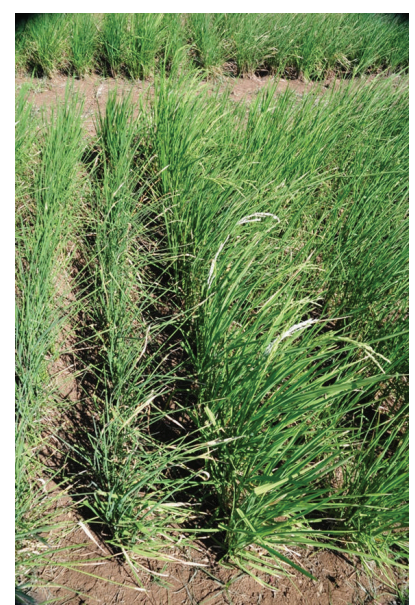

(b)

FIGURE 6: Experiments conducted under (a) lowland drought stress and (b) upland drought stress for screening lines for grain yield under drought.

of lines $(\geq 70 \%)$ showing leaf rolling score of 9 at 10:00 am along with water table and tensiometer readings. A nonstress counterpart must be planted for all stress experiments with similar crop management practices to determine percentage yield reduction under stress. Stress trials can be classified into severe, moderate, or mild stress trials based on the percentage of yield reduction compared with their nonstress counterpart [7]. Under lowland conditions, stress trials showing a yield reduction of $30 \%$ or less are termed mild stress, those with a reduction from $31 \%$ to $65 \%$ are termed moderate stress, and the ones showing a yield reduction above $65 \%$ are referred to as severe stress trials. Under the uplands, due to frequent occurrence of drought with higher severity than in the lowlands, the trials showing a yield reduction of $40 \%$ or less are classified as mild, those with a yield reduction from $41 \%$ to $75 \%$ are classified as moderate, and the ones with a $76 \%$ or higher yield reduction are classified as severe. Data on days to $50 \%$ flowering, plant height, grain yield, biomass, and harvest index can be recorded.

6.2. Genotyping Strategies. The appropriate use of molecular tools is another important aspect of QTL mapping [20]. Rice microsatellite (SSR) markers have been widely used for identification of GY QTLs in several studies [8, 9, 21]. The ease of PCR amplification and electrophoresis makes SSR markers suitable for quick and easy genotyping of large mapping populations to develop a suitable linkage map [20]. The abundant nature of these markers across the rice 
genome allows elaborate coverage of the genome. Moreover, their codominant nature makes them suitable for detecting heterozygotes, making them suitable for genotyping all kinds of mapping populations. Genotyping strategies like selective genotyping (SG), bulk segregant analysis (BSA), and wholegenome scanning (WGS) have been used in different studies to identify QTLs for grain yield under drought (Figure 7).

WGS (Figure 7(a)), although costly, provides an opportunity to identify major-as well as minor-effect QTLs and, at the same time, identify the interaction between different loci. BSA [22] is a technique that involves DNA pooling to identify markers significantly linked to the QTLs. In the case of grain yield under drought, $\sim 4 \%$ of the lines based on phenotypic extremes are identified and used to develop high- and low-yielding bulks [9] that are genotyped along with the parents with all polymorphic markers (Figure 7(b)). The markers having bulk bands corresponding clearly to the parents are considered candidates for full-population genotyping and subsequent identification of QTLs. This technique has been used widely to identify several largeeffect QTLs for grain yield under drought in the background of drought-susceptible varieties such as Swarna, IR64, MTU1010, TDK1, and Sabitri [8, 9, 23-25]. Another traitbased genotyping approach is SG [26]. In SG, a subset of the mapping population constituting $10-15 \%$ of the lines from the phenotypic extremes is selected for genotyping (Figure 7(c)). This minimizes genotyping cost and time consumed and allows the generation of a linkage map and facilitates QTL interaction studies as genotypic data of all markers for the subset of the population are available. $q D T Y_{12.1}$, one of the largest-effect QTLs known for grain yield under drought, was identified through this strategy, in which $12 \%$ of the phenotypic extremes were used for SG [21]. The success of both BSA and SG depends on the precision of phenotyping and the identification strategy of phenotypic extremes [20]. The advancement of genotyping techniques and the availability of high throughput genotyping platforms have allowed rapid genotyping of large populations with high precision. At this point, question can be asked on the relevance of techniques such as BSA and SG for mapping QTLs. Most certainly, these methods still hold their relevance in the field of mapping QTLs. SG can effectively be used along with modern day SNP genotyping platforms to identify QTLs in large populations $(\geq 500$ lines) with reduced costs. Similarly, BSA along with SSR markers can be effectively used for identification of QTLs across multiple mapping populations derived from a common recipient and different donors or vice versa. The QTLs so identified can rapidly be introgressed together to develop drought tolerant versions of popular varieties. While phenotyping of traits is more of a bottleneck in the present day scenario, traits like grain yield can be measured quickly across large populations as compared to other secondary traits. However, the precision of drought stress across the experiments holds the key to success of this strategy. With the advancement of phenotyping techniques in the field, large-scale data sets are likely to be available and these genotyping strategies provide flexibility in terms of genotyping options for the best possible use of large phenotypic datasets.
6.3. Major QTLs for Grain Yield under Drought. Several QTLs for high grain yield under drought have been identified in the recent past using the mapping strategies earlier described (Table 4). Some of these QTLs have shown effects across upland as well as lowland conditions, while some others were found to be specific to ecosystems. Similarly, some QTLs showing effects across multiple genetic backgrounds have been identified, whereas some were found to be more specific to genetic backgrounds. The wide range of effects of these QTLs opens a new door for marker-assisted breeding for drought tolerance. In particular, QTLs that show effects across several genetic backgrounds and/or environments may be used not only for maintenance breeding of existing drought-susceptible popular varieties but also for markerassisted selection for the development of new, high-yielding, drought-tolerant varieties.

$q D T Y_{12.1}$ was the first reported large-effect QTL for grain yield under drought [21]. The SG technique was successfully used in this study for the first time to identify a largeeffect QTL for grain yield under drought. This QTL was identified on a population of 436 random $\mathrm{F}_{3}$-derived lines from a cross between upland rice cultivars Vandana and Way Rarem. Located between RM28048 and RM28166, this QTL explained an $R^{2}$ of $33 \%$ under severe upland drought conditions. Bernier et al. [26] tested the effect of this QTL across 21 experiments conducted at IRRI and in eastern India. The QTL showed consistent performance in both environments with increased effect as severity of stress increased. No effect of the QTL was observed under nonstress condition. The QTL is reported to increase water uptake, which leads to increased yield under drought [27]. While $q D T Y_{12.1}$ was contributed by the susceptible parent Way Rarem, the QTL showed digenic interactions with two other QTLs $\left(q D T Y_{2.3}\right.$ and $q D T Y_{3.2}$ ) contributed by the tolerant parent Vandana [28]. The lines possessing a combination of these three QTLs showed higher advantage in terms of grain yield under drought as compared with those containing individual QTLs. This QTL has also been detected to show a high effect in lowland drought in a $\mathrm{BC}_{1}$-derived population developed from a cross of drought tolerant donor IR74371-46-1-1 and susceptible recipient Sabitri [25]. The QTL spanned between RM28166 and RM28199, explaining a phenotypic variance of $23.8 \%$ [25].

$q D T Y_{2.1}$ and $q D T Y_{3.1}$, two large-effect QTLs affecting grain yield under lowland drought, were identified in a $\mathrm{BC}_{1}$ derived from a cross of high-yielding lowland rice variety Swarna and upland rice variety Apo [9]. The QTLs showed $R^{2}$ values of $16.3 \%$ and $30.7 \%$, respectively, for grain yield under severe lowland drought. BSA was successfully used for the first time to identify such large-effect QTLs for grain yield under drought in this study.

With the successful use of BSA to identify $q D T Y_{3.1}$ and $q D T Y_{2.1}$, large-scale experiments were initiated on $\mathrm{F}_{3}$ derived populations developed from crosses between drought tolerant donor N22 and high-yielding mega varieties Swarna, IR64, and MTU1010 [8]. These experiments resulted in the identification of one of the most consistent QTLs, $q D T Y_{1.1}$, which has shown an effect on grain yield under 


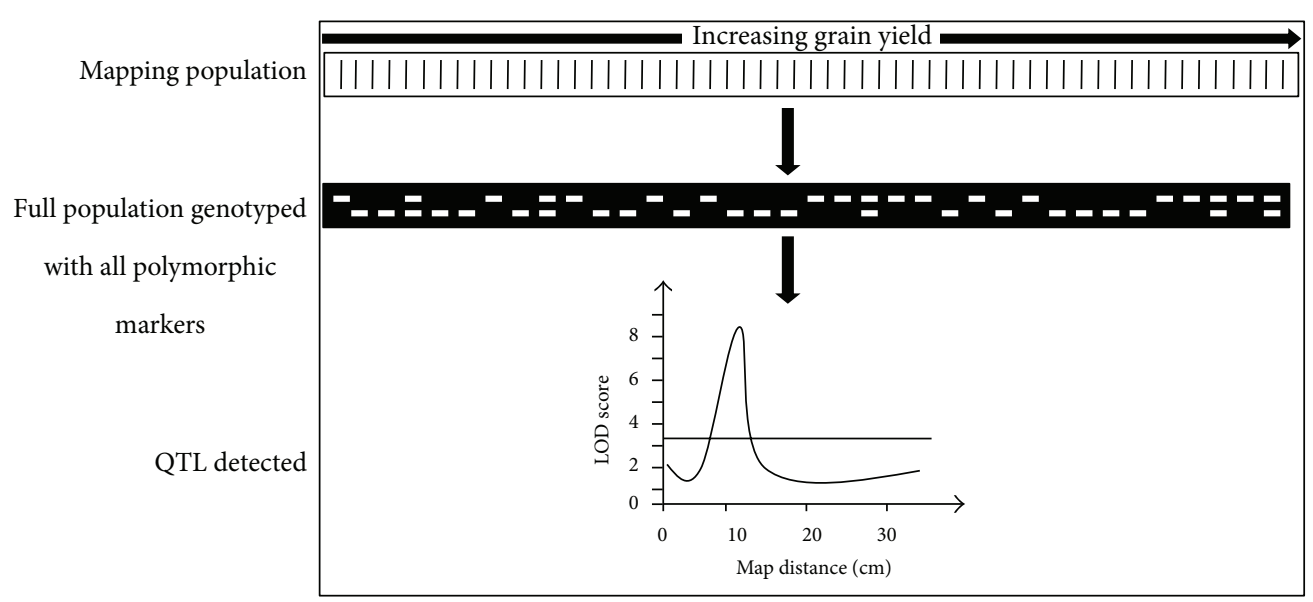

(a)

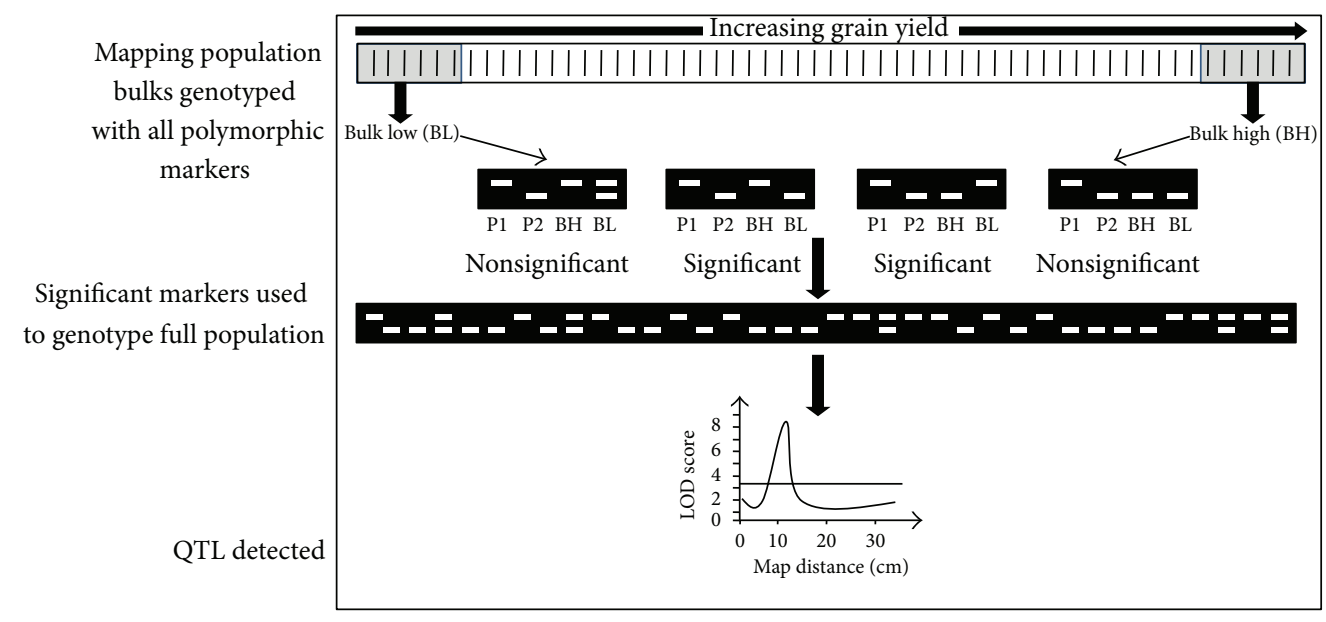

(b)

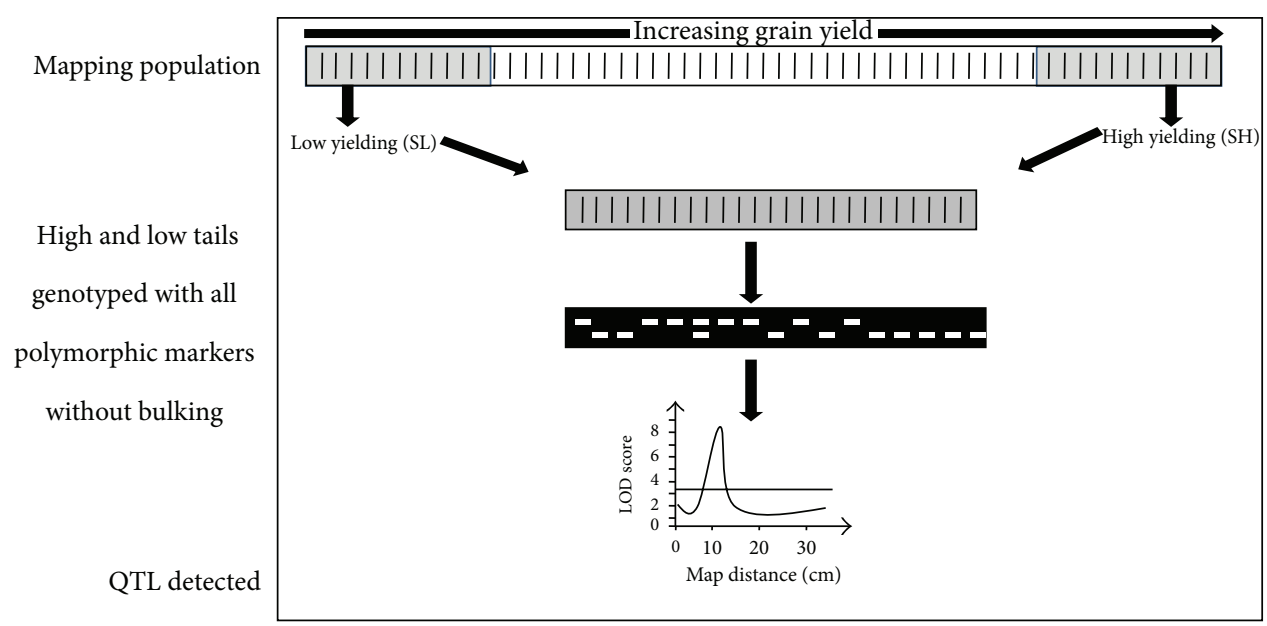

(c)

FIGURE 7: Genotyping strategies used for mapping QTLs for high grain yield under drought by different studies at IRRI: (a) whole-genome scan (WGS), (b) bulk segregant analysis (BSA), and (c) selective genotyping (SG). Source: Kumar et al. [20]. 


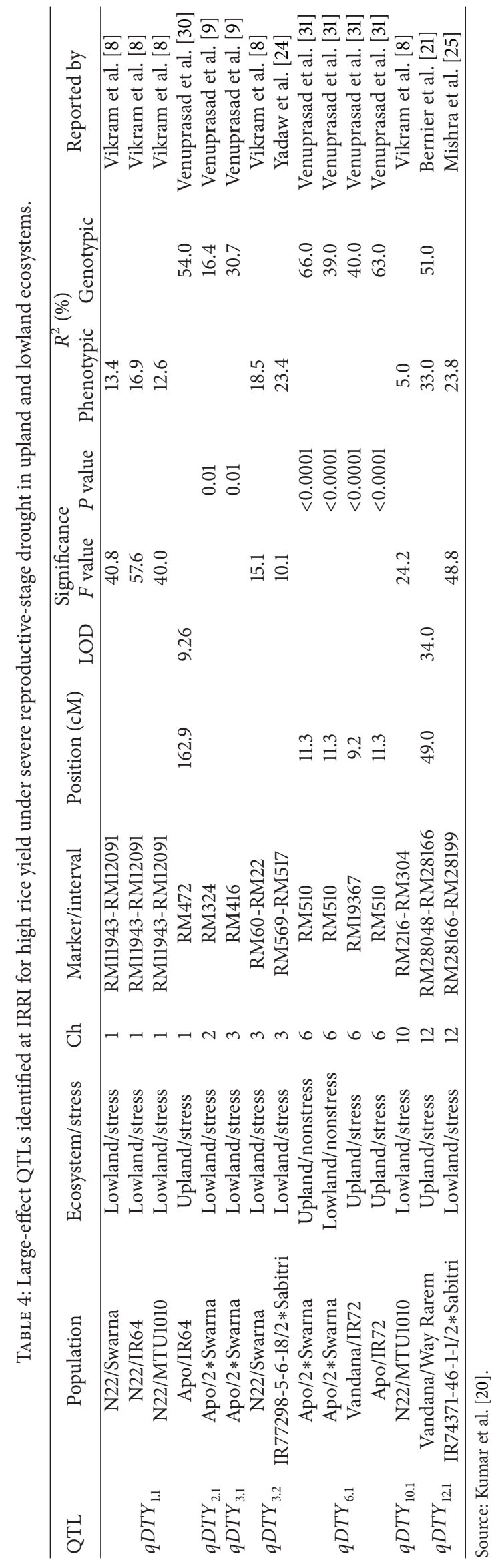


severe lowland drought across the three populations. This QTL showed $R^{2}$ values of $13.4 \%, 16.9 \%$, and $12.6 \%$ across two seasons of screening under severe lowland drought in N22/Swarna, N22/IR64, and N22/MTU1010 populations, respectively. QTLs for grain yield under drought at this locus have also been reported in other populations derived from crosses CT9993-5-10-1-M/IR62266-42-6-2, Apo/IR64, Dhagad deshi/Swarna, and Dhagad deshi/IR64 [23, 29, 30].

$q D T Y_{3.2}$ is another large effect QTL that has shown effect across multiple genetic backgrounds. This QTL co-localizes with $\mathrm{Hd} 9$, a locus which is related to days to flowering (DTF). The locus was first identified to affect DTF in the Vandana/Way Rarem-derived mapping population in a study conducted by Bernier et al. [21]. Later, Vikram et al. [8] reported the effect of this locus on grain yield under drought stress in a N22/Swarna-derived mapping population. The QTL explained a phenotypic variance of 3.2\% across two seasons of testing. In both cases, the QTL was contributed by the early flowering parent and led to a reduction in DTF. Recently, this QTL was reported in a mapping population derived from the cross IR77298-5-6-18 (medium duration)/Sabitri (late duration). In this study, the QTL showed grain yield effect across two seasons of testing at IRRI and in the target environment (Nepal) and explained $23.4 \%$ of the phenotypic variance under severe lowland drought [24]. Surprisingly, the effect of the QTL on grain yield was observed regardless of time of flowering of the lines. The gene content analysis of this QTL also showed the presence of a variety of genes related to plant functions under abiotic stresses, along with the flowering-related gene, $H d 9$. QTLs such as $q D T Y_{3.2}$ can be useful in ecosystems where the effect of drought is very severe and earliness is desirable.

$q D T Y_{6.1}$, a large-effect QTL for grain yield under favourable aerobic and irrigated lowland conditions, was identified in this population [31]. This QTL gave $R^{2}$ values up to $66 \%$ and $39 \%$ under upland and lowland nonstress conditions, respectively.

6.4. Effect of Grain Yield QTLs on Other Traits. Majority of the QTLs affecting grain yield under drought have shown effects on other traits related to yield under drought. There could be three possible reasons for this: (a) the tolerance for drought due to the QTLs affects all these traits, (b) the effects of these QTLs separately on all these traits lead to tolerance for drought, and (c) the effect of the QTLs on grain yield is a combination of both the above mentioned processes. The three phenomena suggest the presence of linkage and/or pleiotropy if the genes affect more than one trait related to plant function under drought. For example, the $q D T Y_{12.1}$ locus showed its effect on almost all traits such as DTF, plant height, biomass, harvest index, drought response index, and panicle number $\mathrm{m}^{-2}$, which affect grain yield under drought [21]. Similarly, the large effect QTL, $q D T Y_{1.1}$ co-localizes with the semi-dwarfing gene $s d 1$. In the study conducted by Vikram et al. [8] for the identification of this QTL, it showed an effect on a variety of traits such as plant height, biomass, and harvest index. $q D T Y_{3.2}$ is another QTL that has shown its effect across varying populations, the colocalization of this
QTL with Hd9 locus leads to its large effect on DTF, which leads to drought escape of lines with this QTL in cases where an early-flowering parent is involved in the development of the population. Systematic studies are underway to break linkage of $q D T Y_{1.1}$ and sd1 to develop high yielding drought tolerant dwarf plant types with $q D T Y_{1.1}$ (IRRI unpublished). However, in case of QTLs related to DTF, the linkage can be utilized either way based on the specific requirements of the target environments. These QTLs can be used beneficially in places where early drought escaping genotypes are needed. However, it has to be taken into account that the extent to which earliness causes decline in grain yield under normal conditions. $q D T Y_{3.2}$ has been a very suitable candidate to be used in such cases. The QTL has consistently been identified across several mapping populations and recombinant lines for these QTLs with late flowering have also been seen. Yadaw et al [24]. also reported this QTL in a population derived from a medium duration line IR77298-5-6-18 and a late duration line Sabitri. No yield decline under nonstress conditions was observed under nonstress conditions in this case. The QTL is now being used to improve Sabitri for drought tolerance and early flowering plants with no yield decline are being obtained (IRRI unpublished). The effect of this QTL has also been seen on traits such as plant height, biomass, and harvest index. A meta-analysis of grain yield QTLs identified during agricultural drought was conducted by Swamy et al. [32]. This study reported a total of 14 meta-QTLs on seven rice chromosomes. A candidate gene content of these metaQTL regions was also reported in this study; it showed the presence of a variety of genes that affect plant function under biotic and abiotic stresses. The study also tested a panel of drought tolerance donors for the presence of these metaQTLs. The survey showed the presence of $q D T Y_{12.1}$ in $85 \%$ of the lines, followed by $q D T Y_{4.1}$ in $79 \%$, and $q D T Y_{1.1}$ in $64 \%$. Comparative genomic analyses of meta-QTL with other cereals were also conducted in this study. They revealed the presence of QTLs for grain yield in the homologous regions of $\mathrm{MQTL}_{1.4}$ and $\mathrm{MQTL}_{3.2}$. The study reported the presence of MQTL 1.4 in maize on chromosome 3 near marker msu2; in wheat, on chromosome $4 \mathrm{~B}$ near marker Rht-b1; and in barley, on chromosome $6 \mathrm{H}$ near marker Bmac0316 while major-effect $\mathrm{MQTL}_{3.2}$ was reported in this study to have a homologous region in maize on chromosome 1 near marker Umc107a. Fine-mapping studies conducted on these QTLs have also shown the presence of multiple sub-QTLs within these regions [33]. The evidence indicates the presence of closely linked genes within these regions, which may have stayed together in the drought tolerance donors during the course of evolution.

\section{Marker-Assisted Breeding for Drought Tolerance}

The identification of drought yield (DTY) QTLs mentioned in the previous sections has opened a way for the development of drought-tolerant versions of popular varieties. Each of the individual DYT QTLs showed a yield advantage of $300-500 \mathrm{~kg} \mathrm{ha}^{-1}$ under moderate to severe drought conditions [20]. This yield advantage needs to be more to make an 


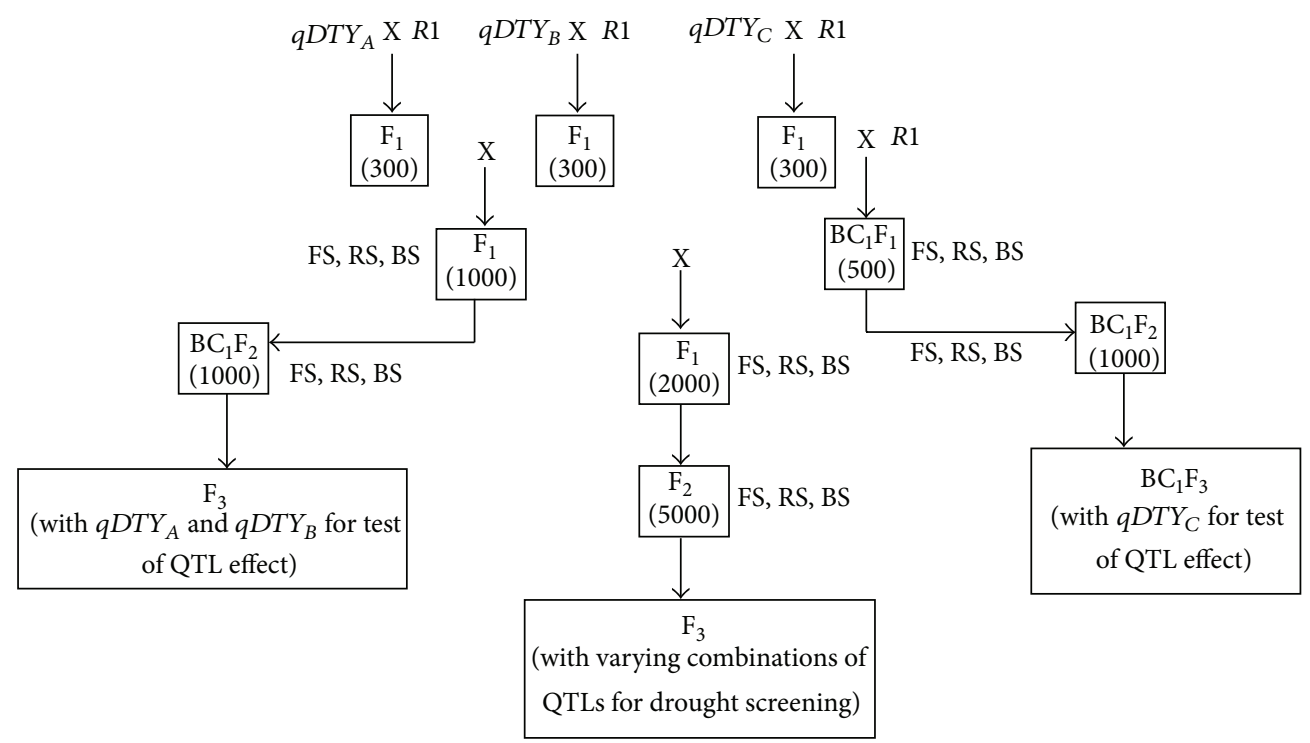

FIGURE 8: Possible marker-assisted backcrossing strategy for pyramiding three large-effect QTLs for drought tolerance in rice from different sources. FS = foreground selection; RS = recombinant selection; BS = background selection. Source: Kumar et al. [20].

impact on a commercial level in farmers' fields. Experiments have shown digenic interactions between DTY QTLs where performance of one QTL is enhanced in the presence of the other [28]. Also, IR64 lines with two and three pyramided QTLs have shown a yield advantage of $1.2-1.5 \mathrm{tha}^{-1}$ over IR64 under moderate to severe drought conditions, while maintaining similar yield potential under normal irrigated conditions [34]. The success of having a combination of QTLs pyramided together has led to the initiation of several other marker-assisted backcrossing (MAB) programs. Three largeeffect QTLs, $q D T Y_{1.1}, q D T Y_{2.1}$, and $q D T Y_{3.1}$, have been introgressed into popular variety Swarna (IRRI, unpublished). Another QTL pyramiding project involving the introgression of $q D T Y_{1.1}, q D T Y_{1.2}, q D T Y_{2.2}$, and $q D T Y_{12.1}$ into IR64 is at its final stages (IRRI, unpublished). Similarly, $q D T Y_{3.1}$, $q D T Y_{6.1}$, and $q D T Y_{6.2}$ are being introgressed into TDK1, a popular variety from Lao PDR (IRRI, unpublished). In all three cases, the respective DTY QTLs are also being combined with Subl to incorporate tolerance for submergence in these varieties, along with drought. Initial results from the screening of Swarna NILs with these QTLs and Sub1 have shown promising results in terms of tolerance for both stresses (IRRI, unpublished). Figure 8 presents the general strategy used for pyramiding the three drought QTLs. QTLs contributed from different sources are combined together through intercrossing and one to two backcrosses are made with the recipient parent based on what is needed to achieve the highest background recovery. Large $F_{1}$ populations are developed at each backcross or intercross based on the number of QTLs involved. Foreground and background selections are made in a specific generation of the pyramiding program to identify lines with all possible combinations of QTLs and the highest background recovery (Figure 7). It has been seen in some cases that lines with a smaller number of QTLs have yielded higher than those with higher number of QTLs, which makes it important to identify lines with all possible combinations of QTLs. For example, in the case of IR64, NILs with $q D T Y_{2.2}$ and $q D T Y_{4.1}$ yielded higher than those with $q D T Y_{2.2}, q D T Y_{4.1}, q D T Y_{9.1}$, and $q D T Y_{10.1}$ [34]. The precise testing of developed lines thus becomes critical in identifying the best combinations of QTLs in the lines.

\section{Conclusion}

Breeding drought-tolerant rice has always been a tough challenge for plant breeders. The use of grain yield as a selection criterion has proved to be a boon in this area of research. Several rice varieties have been released in South and Southeast Asia in recent years. These varieties have shown higher grain yield under drought while maintaining yield potential equal to or more than that of commercial checks in different target environments. The breeding strategy followed at IRRI has also allowed the development of high-yielding varieties tolerant not only of drought but also of biotic stresses such as rice blast and bacterial leaf blight, which are believed to be more prevalent in rainfed areas frequently affected by drought. Several large-effect QTLs for grain yield under drought with effects across genetic backgrounds and environments have been reported in the recent past. These QTLs have allowed the development of drought-tolerant versions of popular mega varieties. The QTLs have also been combined with large QTLs for tolerance for other abiotic stresses such as submergence. Research at IRRI in the recent past has also allowed us to better understand the physiological and molecular mechanisms underlying these QTLs. As development of drought-tolerant NILs for different target environments continues through QTL pyramiding, the NILs not only allow sustainable rice cultivation in areas affected by drought but also open a way to grow rice in new areas. 


\section{Conflict of Interests}

The authors declare that there is no conflict of interests regarding the publication of this paper.

\section{Acknowledgments}

The authors acknowledge the assistance of Ms. M.T. Sta Cruz in drafting the conventional breeding procedure involved in the development of high-yielding drought-tolerant varieties. The authors thank Dr. Casiana Vera Cruz and Ms. Isabelita Oña for the information on bacterial leaf blight and blast screening at IRRI. The authors are grateful to Dr. Andrew Nelson and Ms. Josephine Narciso who provided details about land use patterns in rice. The authors also acknowledge the efforts of Kamoshita et al. 2008 [11] in compiling the review of QTL studies conducted for different traits related to drought tolerance in rice which is provided with this review as supplementary information.

\section{References}

[1] R. Wassmann, S. V. K. Jagadish, K. Sumfleth et al., "Regional vulnerability of climate change impacts on Asian rice production and scope for adaptation," Advances in Agronomy, vol. 102, pp. 91-133, 2009.

[2] D. Molden, K. Frenken, R. Barker et al., "Trends in water and agriculture development," in Water for Food, Water for Life: A Comprehensive Assessment of Water Management in Agriculture, pp. 57-89, International Water Management Institute, Colombo, Sri Lanka; Earthscan, London, UK, 2007.

[3] S. Pandey and H. Bhandari, "Drought economic costs and research implications," in Drought Frontiers in Rice: Crop Improvement for Increased Rainfed Production, pp. 3-17, World Scientific, Singapore; International Rice Research Institute, Los Baños, Philippines, 2008.

[4] S. Pandey, H. Bhandari, R. Sharan, D. Naik, S. K. Taunk, and A. S. R. A.S. Sastri, "Economic costs of drought and rainfed rice farmers' coping mechanisms in Eastern India," Final Project Report, International Rice Research Institute, Los Baños, Philippines, 2005.

[5] J. C. Lanceras, G. Pantuwan, B. Jongdee, and T. Toojinda, "Quantitative trait loci associated with drought tolerance at reproductive stage in rice," Plant Physiology, vol. 135, no. 1, pp. 384-399, 2004.

[6] R. Venuprasad, M. E. Bool, C. O. Dalid, J. Bernier, A. Kumar, and G. N. Atlin, "Genetic loci responding to two cycles of divergent selection for grain yield under drought stress in a rice breeding population," Euphytica, vol. 167, no. 2, pp. 261-269, 2009.

[7] A. Kumar, J. Bernier, S. Verulkar, H. R. Lafitte, and G. N. Atlin, "Breeding for drought tolerance: direct selection for yield, response to selection and use of drought-tolerant donors in upland and lowland-adapted populations," Field Crops Research, vol. 107, no. 3, pp. 221-231, 2008.

[8] P. Vikram, B. P. M. Swamy, S. Dixit et al., "qDTY ${ }_{1.1}$, a major QTL for rice grain yield under reproductive-stage drought stress with a consistent effect in multiple elite genetic backgrounds," $B M C$ Genetics, vol. 12, article 89, 2011.

[9] R. Venuprasad, C. O. Dalid, M. del Valle et al., "Identification and characterization of large-effect quantitative trait loci for grain yield under lowland drought stress in rice using bulksegregant analysis," Theoretical and Applied Genetics, vol. 120, no. 1, pp. 177-190, 2009.

[10] S. Fukai and M. Cooper, "Development of drought-resistant cultivars using physio-morphological traits in rice," Field Crops Research, vol. 40, no. 2, pp. 67-86, 1995.

[11] A. Kamoshita, R. C. Babu, N. M. Boopathi, and S. Fukai, "Phenotypic and genotypic analysis of drought-resistance traits for development of rice cultivars adapted to rainfed environments," Field Crops Research, vol. 109, no. 1-3, pp. 1-23, 2008.

[12] M. Ashraf, "Inducing drought tolerance in plants: recent advances," Biotechnology Advances, vol. 28, no. 1, pp. 169-183, 2010.

[13] A. Kumar, S. Verulkar, S. Dixit et al., "Yield and yield-attributing traits of rice (Oryza sativa L.) under lowland drought and suitability of early vigor as a selection criterion," Field Crops Research, vol. 114, no. 1, pp. 99-107, 2009.

[14] R. Venuprasad, H. R. Lafitte, and G. N. Atlin, "Response to direct selection for grain yield under drought stress in rice," Crop Science, vol. 47, no. 1, pp. 285-293, 2007.

[15] S. B. Verulkar, N. P. Mandal, J. L. Dwivedi et al., "Breeding resilient and productive genotypes adapted to drought-prone rainfed ecosystem of India," Field Crops Research, vol. 117, no. 2-3, pp. 197-208, 2010.

[16] G. N. Atlin and K. J. Frey, "Selecting oat lines for yield in lowproductivity environments," Crop Science, vol. 30, no. 3, pp. 556561, 1990.

[17] R. Serraj, A. Kumar, K. L. McNally et al., "Improvement of drought resistance in rice," Advances in Agronomy, vol. 103, pp. 41-99, 2009.

[18] R. Venuprasad, M. T. Sta Cruz, M. Amante, R. Magbanua, A. Kumar, and G. N. Atlin, "Response to two cycles of divergent selection for grain yield under drought stress in four rice breeding populations," Field Crops Research, vol. 107, no. 3, pp. 232-244, 2008.

[19] Standard Evaluation System for Rice, International Rice Research Institute, 4th edition, 1996.

[20] A. Kumar, S. Dixit, and A. Henry, "Marker-assisted introgression of major QTLs for grain yield under drought in rice," in Translational Genomics for Crop Breeding: Abiotic Stress, Yield and Quality, Volume 2, R. K. Varshney and R. Tuberosa, Eds., 1st edition, 2013.

[21] J. Bernier, A. Kumar, V. Ramaiah, D. Spaner, and G. Atlin, "A large-effect QTL for grain yield under reproductive-stage drought stress in upland rice," Crop Science, vol. 47, no. 2, pp. 507-518, 2007.

[22] R. W. Michelmore, I. Paran, and R. V. Kesseli, "Identification of markers linked to disease-resistance genes by bulked segregant analysis: a rapid method to detect markers in specific genomic regions by using segregating populations," Proceedings of the National Academy of Sciences of the United States of America, vol. 88, no. 21, pp. 9828-9832, 1991.

[23] K. H. Ghimire, L. A. Quiatchon, P. Vikram et al., "Identification and mapping of a QTL ( qDTY $\left._{1.1}\right)$ with a consistent effect on grain yield under drought," Field Crops Research, vol. 131, pp. 88-96, 2012.

[24] R. B. Yadaw, S. Dixit, A. Raman et al., "A QTL for high grain yield under lowland drought in the background of popular rice variety Sabitri from Nepal," Field Crops Research, vol. 144, pp. 281-287, 2013. 
[25] K. K. Mishra, P. Vikram, R. B. Yadaw et al., "qDTY ${ }_{12.1}$ : a locus with a consistent effect on grain yield under drought in rice," BMC Genetics, vol. 14, article 12, 2013.

[26] R. J. Lebowitz, M. Soller, and J. S. Beckmann, "Trait-based analyses for the detection of linkage between marker loci and quantitative trait loci in crosses between inbred lines," Theoretical and Applied Genetics, vol. 73, no. 4, pp. 556-562, 1987.

[27] J. Bernier, R. Serraj, A. Kumar et al., "The large-effect droughtresistance QTL qtl12.1 increases water uptake in upland rice," Field Crops Research, vol. 110, no. 2, pp. 139-146, 2009.

[28] S. Dixit, B. P. M. Swamy, P. Vikram et al., "Increased drought tolerance and wider adaptability of $\mathrm{qDTY}_{12.1}$ conferred by its interaction with $\mathrm{qDTY}_{2.3}$ and $\mathrm{qDTY}_{3.2}$," Molecular Breeding, vol. 30, no. 4, pp. 1767-1779, 2012.

[29] R. Kumar, R. Venuprasad, and G. N. Atlin, "Genetic analysis of rainfed lowland rice drought tolerance under naturallyoccurring stress in eastern India: heritability and QTL effects," Field Crops Research, vol. 103, no. 1, pp. 42-52, 2007.

[30] R. Venuprasad, M. E. Bool, L. Quiatchon, M. T. Sta Cruz, M. Amante, and G. N. Atlin, "A large-effect QTL for rice grain yield under upland drought stress on chromosome 1," Molecular Breeding, vol. 30, pp. 535-547, 2012.

[31] R. Venuprasad, M. E. Bool, L. Quiatchon, and G. N. Atlin, “A QTL for rice grain yield in aerobic environments with large effects in three genetic backgrounds," Theoretical and Applied Genetics, vol. 124, no. 2, pp. 323-332, 2012.

[32] B. M. Swamy, P. Vikram, S. Dixit, H. Ahmed, and A. Kumar, "Meta-analysis of grain yield QTL identified during agricultural drought in grasses showed consensus," BMC Genomics, vol. 12, article 319, 2011.

[33] S. Dixit, B. P. M. Swamy, P. Vikram et al., "Fine mapping of QTLs for rice grain yield under drought reveals sub-QTLs conferring a response to variable drought severities," Theoretical and Applied Genetics, vol. 125, no. 1, pp. 155-169, 2012.

[34] B. P. M. Swamy, H. U. Ahmed, A. Henry et al., "Genetic, physiological, and gene expression analyses reveal that multiple QTL enhance yield of rice mega-variety IR64 under drought," PLoS ONE, vol. 8, no. 5, Article ID e62795, 2013.

[35] K. A. Steele, A. H. Price, H. E. Shashidhar, and J. R. Witcombe, "Marker-assisted selection to introgress rice QTLs controlling root traits into an Indian upland rice variety," Theoretical and Applied Genetics, vol. 112, no. 2, pp. 208-221, 2006.

[36] K. A. Steele, D. S. Virk, R. Kumar, S. C. Prasad, and J. R. Witcombe, "Field evaluation of upland rice lines selected for QTLs controlling root traits," Field Crops Research, vol. 101, no. 2, pp. 180-186, 2007.

[37] K. Steele, Novel Upland Rice Variety Bred Using Marker-Assisted Selection and Client Oriented Breeding Released in Jharkhand, India, Bangor University, 2009.

[38] R. Hijmans, “The where and how of rice," Rice Today, vol. 6, pp. 19-20, 2007. 


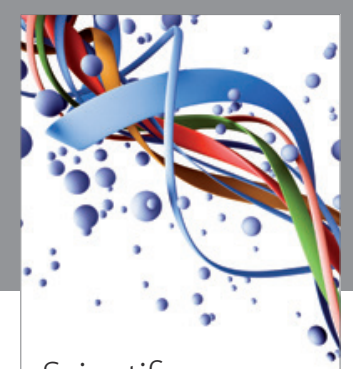

Scientifica
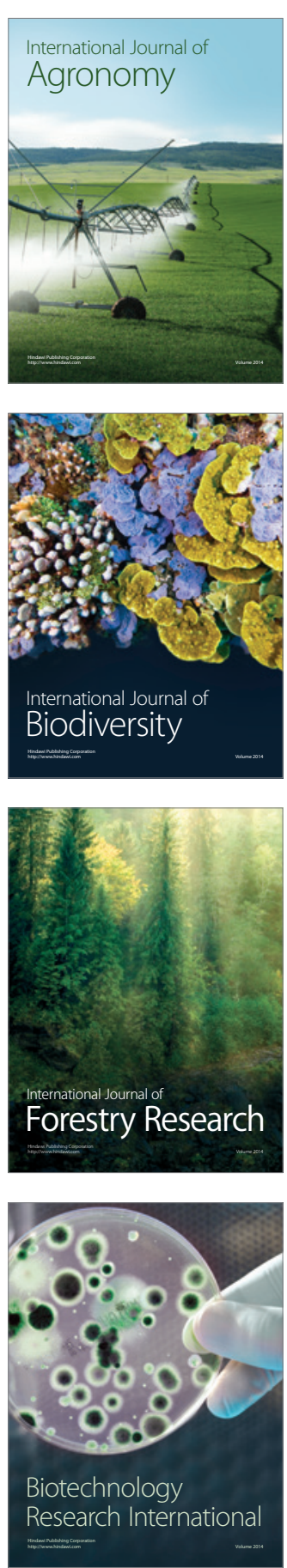
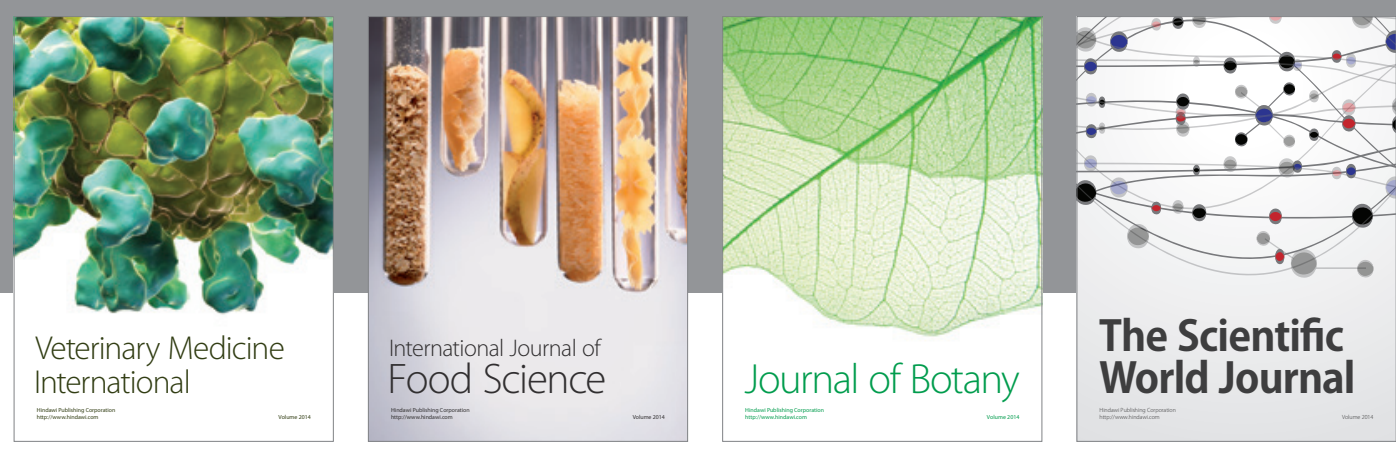

The Scientific World Journal
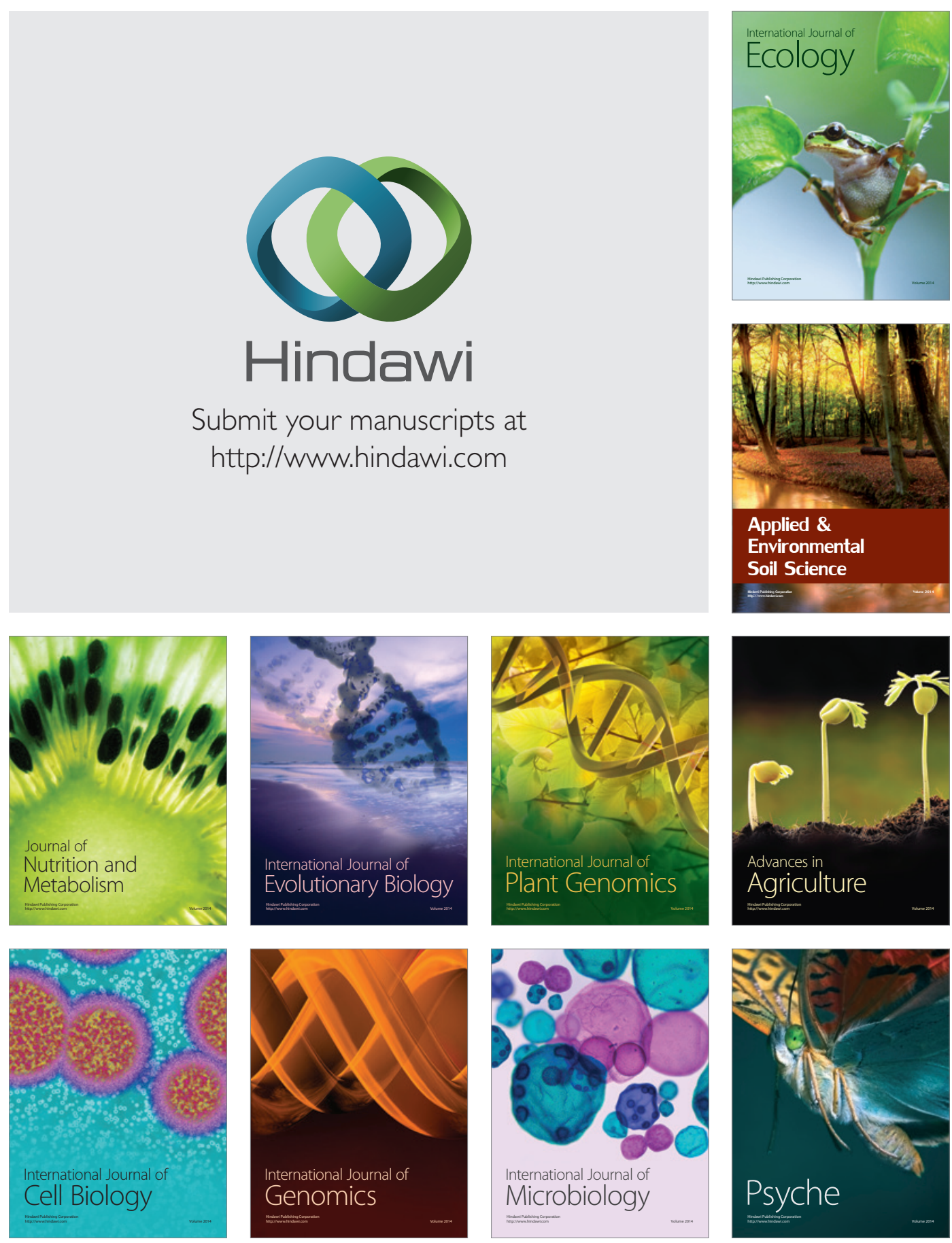